\title{
OPEN Sugary drink consumption and risk of kidney and bladder cancer in Japanese adults
}

\author{
ChiYan Leung ${ }^{1}$, Sarah Krull Abe ${ }^{2}$, Norie Sawada ${ }^{2}$, Junko Ishihara ${ }^{3}$, Ribeka Takachi ${ }^{4}$, \\ TaikiYamaji ${ }^{2}$, Motoki Iwasaki², Masahiro Hashizume ${ }^{1}$, Manami Inoue $^{2,5 \bowtie}$ \& \\ Shoichiro Tsugane ${ }^{2}$
}

Globally, sugary drinks are widely consumed, however, few epidemiologic studies have investigated the association between sugary drink consumption and risk of kidney and bladder cancer. We examined the association of sugary drinks with risk of kidney and bladder cancer in 73,024 participants from the Japan Public Health Center-based Prospective Study who reported no history of cancer. Sugary drink consumption was assessed using a validated food frequency questionnaire at study baseline (1995-1999). Individuals were followed to December 31, 2013. Multivariable Cox proportional hazards regression models were used to calculate hazard ratios (HR) and $95 \%$ confidence intervals (Cls). During 1,069,815 person years of follow-up, 169 kidney cancer and 297 bladder cancer cases were documented. After adjusting for potential confounders, no greater risk of kidney and bladder cancer was observed. However, sugary drink consumption was positively associated with the risk of kidney cancer (HR for $100 \mathrm{ml} /$ day increase in consumption was 1.11 [95\% Cl 1.01-1.22]) and bladder cancer (HR for $100 \mathrm{ml} / \mathrm{d}$ increase in consumption was 1.11 [95\% Cl 1.01-1.22]) among women after exclusion of cases diagnosed in the first three years of follow-up. In this large prospective cohort, consumption of sugary drinks was significantly associated with a small increase in hazard ratio for kidney and bladder cancer among women after exclusion of cases diagnosed within the first three years.

Globally, the age-standardized incidence rates of kidney and bladder cancer have increased by $29 \%$ and $4 \%$ between 1990 and 2019, respectively ${ }^{1}$. In 2018, 403,262 new cases of kidney cancer and 549,393 new cases of bladder cancer were diagnosed ${ }^{2}$, accounting for the 16th- and 12th-most common cancers, respectively ${ }^{2}$. Despite these increasing trends, few modifiable lifestyle risk factors for kidney and bladder cancer have been identified, and no dietary factor has been clearly linked to risk $^{3,4}$.

Sugary drinks, which are widely consumed globally, have been associated with overall and breast cancer incidence ${ }^{5}$ and have gained increasing interest in cancer development. The high fructose content of sugary drinks has been associated with postprandial hyperuricemia, which in turn contributes to a decline in renal function ${ }^{6}$. A study in more than one million participants aged 40 years or older showed a positive association between reduced kidney function $($ GFR $<30)$ and risk of kidney cancer ${ }^{7}$. In addition, previous studies found that the consumption of sugary drinks was associated with diabetes, hypertension, and obesity ${ }^{8}$ which are known risk factors for kidney cancer ${ }^{3,9}$. Although there are several plausible mechanisms for how sugary drink consumption could lead to urinary bladder carcinogenesis ${ }^{10,11}$, their relative strengths have not been fully elucidated. On one hand, the urogenous hypothesis states that higher amounts of fluid intake may reduce bladder cancer risk by increasing micturition frequency and reducing the exposure time of the bladder to carcinogenic substances ${ }^{10}$, while on the other, contact of potential carcinogens in cola-type beverages with the bladder urothelium, such as 4-methylimidazole (IARC Group 2B carcinogen), may increase risk of bladder cancer ${ }^{11}$. In addition, sugary drinks have a high glycemic index, which was associated with bladder cancer risk in a meta-analysis of four studies (pooled odds ratio $1.25,95 \%$ CI $\left.1.11-1.41, I^{2} 0.0 \%\right)^{12}$.

\footnotetext{
${ }^{1}$ Department of Global Health Policy, Graduate School of Medicine, The University of Tokyo, Tokyo, Japan. ${ }^{2}$ Epidemiology and Prevention Group, Division of Prevention, Center for Public Health Sciences, National Cancer Center, 5-1-1 Tsukiji, Chuo-ku, Tokyo 104-0045, Japan. ${ }^{3}$ Department of Food and Life Science, Azabu University, Kanagawa, Japan. ${ }^{4}$ Department of Food Science and Nutrition, Graduate School of Humanities and Sciences, Nara Women's University, Nara, Japan. ${ }^{5}$ Department of Cancer Epidemiology, Graduate School of Medicine, The University of Tokyo, Tokyo, Japan. ${ }^{\boxplus e m a i l: ~ m n m i n o v e @ n c c . g o . j p ~}$
} 
To date, evidence regarding the association of sugary drink consumption with kidney and bladder cancer is sparse, and findings are inconsistent ${ }^{13-16}$. To our knowledge, no study has investigated potential mediators and modifiers of the albeit putative effect. In addition, there are concerns regarding reverse causality in prior literature, wherein early symptoms of undiagnosed cancer may affect sugary drink consumption habits. To fill this knowledge gap, we conducted a large prospective cohort study to investigate the association between sugary drink consumption and subsequent risk of kidney and bladder cancer over a 16-year median follow-up. We also examined reverse causation and potential effects of mediation and modification by the prespecified variables of body mass index (BMI), diabetes, and hypertension.

\section{Methods}

Study population. The Japan Public Health Center-based Prospective Study (JPHC Study) included 140,420 Japanese aged 40-69 years at the inception of the study, recruited from the general population in 1990 to 1994 in 11 prefectural public health center (PHC) areas. In 1990, 61,595 individuals were enrolled in Cohort I (Akita, Iwate, Nagano, Okinawa-Chubu, and Tokyo) and 78,825 were enrolled in 1993 in Cohort II (Ibaraki, Kochi, Niigata, Nagasaki, Osaka, and Okinawa-Miyako). At enrollment, participants answered self-administered food frequency questionnaires (FFQs) and provided information on their medical history and lifestyle. An expanded FFQ was sent at five-year follow-up to collect updated dietary information. For the current analysis, we used data from the five-year survey as baseline because it included a more comprehensive assessment of sugary beverage intake (eight sugary drink items) than that used at enrollment (two sugary drink items). Because of a lack of cancer incidence data, 7097 participants living in the Tokyo area were excluded. We further excluded non-Japanese nationals, $(n=51)$, pre-commencement migration $(n=188)$, those who had incorrect birth date $(n=7)$, duplicate registration $(n=10)$, or died or moved away from the study area before baseline of this analysis $(n=12,166)$. Additionally, we excluded those with cancer $(n=4,103)$, had not completed the follow-up questionnaires $(n=22,704)$, had the highest or lowest 2.5 percentile of calorie intake $(<993$ or $>4204 \mathrm{kcal} /$ day for men and $<837$ or $>3683 \mathrm{kcal} / \mathrm{d}$ for women $)(\mathrm{n}=5675)$, or had missing covariate information $(\mathrm{n}=15,395)$. Finally, this resulted in the inclusion of 73,024 participants (33,094 men and 39,930 women). Table S1 presents key characteristics of included and excluded participants. Enrolled individuals were informed of the objectives of the study. Informed consent was obtained from all participants when completing the baseline questionnaire. The National Cancer Center Japan institutional review board has approved this study (approval number: 2001-021).

Dietary assessment. The 5-year follow-up FFQ with 138 food and beverage items was validated using 14- or 28-day dietary records. Validation of the questionnaire has been published elsewhere ${ }^{17,18}$. In the present analysis, we defined sugary drinks as beverages that contain caloric sweeteners, which included $100 \%$ fruit juices (orange juice and apple juice), beta-carotene-fortified beverages, calcium-fortified beverages, canned coffee, carbonated beverages, lactic acid bacteria beverages, and vitamin-fortified beverages ${ }^{19,20}$. Lactic acid bacteria beverages are dairy products containing probiotics ${ }^{21}$. Sugar-sweetened tea and artificially sweetened beverages were not included in the FFQ. In the FFQ, nine options from "Never", "1-2 times/week", "3-4 times/week", "5-6 times/ week", "1 cup/day”, "2-3 cups/day", "4-6 cups/day”, "7-9 cups/day”, to "10 or more cups/day" were used to assess how often participants consumed sugary drinks. Standard portion size was defined as $250 \mathrm{ml}$ for carbonated beverages and canned coffee; $200 \mathrm{ml}$ for $100 \%$ fruit juices (orange juice and apple juice), beta-carotene fortified beverages, calcium-fortified beverages, and vitamin-fortified beverages; and $65 \mathrm{ml}$ for lactic acid bacteria beverages. In the cohort, Spearman's coefficients for validity were 0.32 (men) and 0.21 (women). Reproducibility of the FFQ was assessed through two FFQs one year apart, showing Spearman's coefficients for reproducibility of 0.63 (men) and 0.56 (women) ${ }^{19,20}$.

Case ascertainment. The occurrence of incident cancer was identified through record linkage to population cancer registries and by active patient notification from hospitals in the study areas, consisting of a supplement to death certificate files. Among kidney cancer cases, $6.9 \%$ were notified by death certificate (DCN), and $4.6 \%$ were ascertained by Death Certificate Only (DCO). For bladder cancer cases, DCN was $4.9 \%$ and DCO was $2.3 \%$. The Third Edition of the International Classification of Disease for Oncology (ICD-10) was used to identify kidney cancer (C64) and bladder cancer (C67) 22 .

Statistical analysis. Individuals were followed and person-years for each individual were calculated from the date of completion of the questionnaires in 1995 and 1998-1999 to the date of moving out of the study areas, death, or the study end date (31 December 2013; except Osaka, 31 December 2012), whichever occurred first. We categorized participants according to total daily sugary drink consumption (ml) rather than frequency owing to the different standard portion sizes for beverage items. Sugary drink consumers were divided into two categories based on sex-specific median consumption level $(<254 \mathrm{ml} /$ day and $\geq 254 \mathrm{ml} /$ day for $\mathrm{men}$, and $<134 \mathrm{ml} /$ day and $\geq 134 \mathrm{ml} /$ day for women). The choice of median values was based on the consumption distributions of the present study, which allowed us to balance sample size between groups. Non-consumers were used as the reference category. Hazard ratios (HR) with 95\% confidence intervals (CIs) over intake categories and by each $100-\mathrm{ml}$ increase were evaluated using Cox proportional hazards regression models stratified by sex and adjusted for age (year, continuous); study area (10 PHC areas); history of diabetes (yes or no $)^{23-25}$; body mass index (BMI) $(<18.5$, $18.5-<25,25-<30$, or $30-<45)^{26}$; hypertension history (yes or no $)^{27}$; smoking status and intensity (never; former; current: $<20$ cigarettes/day; current: $\geq 20$ cigarettes/day) ${ }^{28}$; consumption of alcohol (ethanol gram/week,

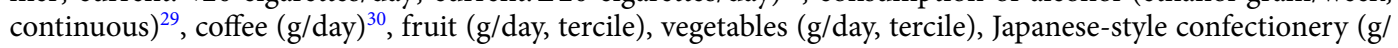
day, tercile), chocolate (g/day, tercile), biscuits (g/day, tercile), and cake (g/day, tercile); and physical activities (metabolic equivalent task, hours/day, tercile); height ( $\mathrm{cm}$, tercile); and total calorie intake (kcal/day, continu- 
ous). Figure S1 presents the directed acyclic graph used to determine the minimal adjustment set. The association of sugary drink consumption with risk of kidney and bladder cancer was also assessed across sex.

In sensitivity analysis, cancer cases occurring in the first three years of follow-up were excluded to minimize reverse causality. To estimate the degree to which the associations were mediated by obesity, diabetes, and hypertension, we repeated the analysis without adjustment for BMI, history of diabetes, and history of hypertension. In addition, subgroup analysis was performed to examine whether the associations between consumption of sugary drinks and risk of kidney and bladder cancer were modified by body mass index $\left(\mathrm{kg} / \mathrm{m}^{2} ;<25\right.$ or $\left.\geq 25\right)$, history of diabetes (yes or no), and history of hypertension (yes or no). Likelihood ratio tests were used to test for interactions. We used Schoenfeld residuals to examine the proportionality of hazards for those covariates included in the Cox model, and no violation was found. In each model, a linear trend across categories was also tested using the median intake of each category as a continuous variable. To examine potential nonlinear associations, we conducted a likelihood ratio test in order to compare the model with linear and cubic spline terms for sugary drink consumption to the model only with linear term. Restricted cubic splines with five knots were used (placed at the 5th, 27.5th, 50th, 72.5th, and 95th percentiles) ${ }^{31}$. All statistical analyses were conducted using Stata STATA 14 (Stata Corp., College Station, TX, USA), with $P<0.05$ considered statistically significant.

Ethics approval and consent to participate. The study was conducted in compliance with the provisions of the Declaration of Helsinki. The study protocol was approved by the Institutional Review Board of the National Cancer Center, Japan. The participants were informed of the study objectives, and those who completed the survey questionnaire were regarded as consenting to participation.

\section{Results}

During a median follow-up of 15.8 years (range 0 to 18.9 years; $1,069,815$ person-years) among participants in the JPHC Study, 169 kidney and 297 bladder cancers were ascertained (Fig. 1). Participant baseline characteristics according to sugary drink consumption level are presented in Table 1. Compared with non-consumers, sugary drink consumers tended to be current smokers, younger, and with a higher consumption of total energy. In contrast, non-consumers were more likely to consume alcohol and have a past history of hypertension and diabetes. Women who consumed greater amounts of sugary drinks had a higher BMI than non-consumers.

In minimally adjusted and multivariable analyses, there was no association of sugary drink consumption with risk of kidney cancer in men and women combined (Table 2). A similar lack of association was observed when we analyzed men and women separately. Compared with non-consumers, men with a consumption of $\geq 254 \mathrm{ml}$ per day of sugary drinks had a HR of $0.89(95 \%$ CI $0.49-1.62, P=0.71)$ for kidney cancer. In women, the multivariable HR for kidney cancer among those who had consumed $\geq 134 \mathrm{ml}$ per day was 0.93 (95\% CI 0.43-2.03, $P=0.86$ ). Further, no association was found between sugary drink consumption and bladder cancer among both women and men (Table 2). Compared to non-consumers, the multivariable-adjusted HR for bladder cancer among men was 0.73 (95\% CI $0.49-1.08, P=0.11$ ) for those who consumed $\geq 254 \mathrm{ml}$ per day. Consumption was not associated with increased bladder cancer risk among women (non-consumers vs. $\geq 134 \mathrm{ml}$ per day; HR, $0.95 ; 95 \% \mathrm{CI}$ $0.45-2.03, P=0.90)$. In mediation analysis, the results remained unchanged after exclusion of BMI and histories of diabetes and hypertension from the model.

To minimize reverse causation, we excluded 23 kidney cancer and 32 urinary bladder cancer cases that were diagnosed within the first three years of follow-up. In categorical analysis, no association with kidney and bladder cancer was found. In continuous analyses, consumption of sugary drinks was marginally positively associated with the risk of kidney cancer (HR for $100 \mathrm{ml}$ per day increase, 1.11; 95\% CI 1.01-1.22, $P=0.03$ ) and bladder cancer (HR for $100 \mathrm{ml}$ per day increase, 1.11; 95\% CI 1.01-1.22, $P=0.03$ ) among women (Table 2). Restricted cubic spline regression analyses showed that the relations between sugary drink consumption and risk of kidney (men, $P=0.13$; women, $P=0.18$ ) and bladder cancer (men, $P=0.67$; women, $P=0.06$ ) were consistent with linear associations. No differences were observed in the associations of sugary drink consumption with risk of kidney and bladder cancer when stratified by BMI and histories of diabetes and hypertension (Table 3 ).

\section{Discussion}

In this large population-based prospective cohort of 73,024 participants with a 15.8-year median follow-up, we found null associations between sugary drink consumption and risk of kidney and bladder cancers. However, our results suggest that higher sugary drink consumption was associated with a greater risk of kidney and bladder cancers among women after excluding kidney and bladder cancer cases diagnosed within three years after baseline assessment.

Sugary drink consumption has been hypothesized to increase kidney cancer risk ${ }^{7,32-39}$, but two previous studies did not find positive associations ${ }^{13,14}$. In a pooled analysis involving 13 prospective studies, no association was observed between soda consumption and renal cell cancer (pooled multivariable relative risk increment of $355 \mathrm{ml}$ per day $=1.02 ; 95 \%$ CI 0.9 to 1.06$)^{14}$. Consistently, higher consumption of sugar-sweetened soft drinks was not associated with kidney cancer risk in the Melbourne Collaborative Cohort Study $(\geq 1$ cup/day vs $<1$ cup/month; HR 1.32; $95 \%$ CI 0.79 to 2.19$)^{13}$. In the current study, the null association of sugary drinks with risk of kidney cancer in the primary analysis accords with results from previous studies ${ }^{13,14}$. However, sugary drink consumption at baseline may have been changed or under-reported for participants with obesity, hypertension, or diabetes, which may bias the associations downward. To address this, we adjusted these covariates in our multivariable models and assessed the association after exclusion of participants diagnosed with cancer during the first three years. After exclusion, positive associations between sugary drink consumption and risk of kidney and bladder cancer were observed in women. Of note, this exclusion may have reduced the statistical power of our analyses. Reverse causation was also assessed in the Melbourne Collaborative Cohort Study, but the results 


\section{Individuals enrolled in cohort I and II ( $n=140420)$}

\begin{tabular}{l|l} 
& Excluded $(n=19519)$ \\
Participants from Tokyo $(n=7097)$ \\
Non-Japanese nationality $(n=51)$ \\
Pre-commencement migration $(n=188)$ \\
Incorrect birth date $(n=7)$ \\
Duplicate registration $(n=10)$ \\
Moved out or died before Qo5 $(n=12166)$
\end{tabular}

Individuals assessed for eligibility $(n=120$ 901)

$\begin{aligned} & \text { Individuals responding to questionnaire }(n=98197) \\ & \text { Response rate: } 81 \%\end{aligned} \mid \begin{aligned} & \text { Without responses }(n=22704) \\ & \text { Excluded }(n=25173) \\ & \text { Cancer history }(n=4103) \\ & \text { Outlier with implausible energy intake }(n=5675) \\ & \text { Missing information }(n=15395)\end{aligned}$

Individuals included in analysis $(n=73024)$

\section{8 years of median follow-up 1069815 person-years}

Kidney cancer $(n=169)$, bladder cancer $(n=297)$

Figure 1. Participant flow chart.

remained nonsignificant after excluding cases diagnosed in the first 2 years. The lack of significance in the Melbourne study might have been due to the limited number of cases (146 kidney cancer cases before exclusion). Nevertheless, the potential influence of changes in diet and lifestyle in the present study cannot be eliminated completely, and thus risk may be underestimated.

Two previous studies on the association of sugary drink consumption with bladder cancer have been conducted, albeit with conflicting results. In the European Prospective Investigation into Cancer and Nutrition (EPIC), increased consumption of soft drinks was weakly associated with an increased risk of urothelial cell carcinoma (HR for $100 \mathrm{ml}$ incremental, 1.06; 95\% CI 1.01 to 1.12$)^{15}$. On the other hand, the Health Professionals Follow-up Study (HPFS) reported a null association of bladder cancer risk with consumption of soda and lemonade (HR for $240 \mathrm{ml}$ incremental, $0.99 ; 95 \%$ CI 0.90 to 1.08$)^{16}$. Reverse causation was not assessed in these two studies. Our findings in the primary analysis did not support the association of sugary drinks with bladder cancer risk. The length of time that carcinogens are in contact with urothelial cells of the bladder has been postulated to be associated with bladder cancer risk and may explain the null association ${ }^{10}$. Higher fluid consumption increases urination volume and frequency, thereby reducing contact time and likely counterbalancing the 


\begin{tabular}{|c|c|c|c|}
\hline Characteristic & \multicolumn{3}{|c|}{ Category of sugary drink consumption } \\
\hline \multicolumn{4}{|l|}{ Men } \\
\hline Intake category, ml/day & Non-consumers & $>0-254$ & $\geq 254$ \\
\hline Number & 4363 & 14,366 & 14,365 \\
\hline Age, mean (SD), year & $58.2(7.9)$ & $57.0(7.6)$ & $55.2(7.6)$ \\
\hline Physical activity, mean (SD), METS-h/day & $31.7(6.4)$ & $32.7(6.8)$ & $33.3(6.9)$ \\
\hline BMI, mean (SD), kg/m² & $23.6(2.9)$ & $23.7(2.8)$ & $23.6(2.9)$ \\
\hline Past history of hypertension, \% & 18.4 & 15.9 & 12.4 \\
\hline Past history of diabetes, $\%$ & 14.9 & 9.7 & 6.4 \\
\hline Current smoker, \% & 42.4 & 43.4 & 52.8 \\
\hline Current alcohol consumption, \% & 75.5 & 75.6 & 68.7 \\
\hline Total energy, mean (SD), kcal/day & $1958.4(567.8)$ & $2204.7(628.4)$ & $2222.9(644.3)$ \\
\hline Vegetables, mean (SD), g/day & $139.0(92.1)$ & $133.9(85.8)$ & $125.3(76.3)$ \\
\hline Fruit, mean (SD), g/day & $70.3(70.1)$ & $83.5(68.7)$ & $101.2(78.6)$ \\
\hline Coffee consumption, mean (SD), g/day & $127.5(196.1)$ & $121.7(188.3)$ & $128.8(181.8)$ \\
\hline \multicolumn{4}{|l|}{ Women } \\
\hline Intake category, $\mathrm{ml} /$ day & Non-consumers & $>0-134$ & $\geq 134$ \\
\hline Number & 6380 & 16,775 & 16,775 \\
\hline Age, mean (SD), year & $57.0(8.0)$ & $56.8(7.7)$ & $56.2(7.7)$ \\
\hline Physical activity, mean (SD), METS-h/day & $31.6(5.6)$ & $32.1(5.7)$ & $32.3(5.9)$ \\
\hline BMI, mean (SD), kg/m² & $23.3(3.2)$ & $23.5(3.1)$ & $23.6(3.2)$ \\
\hline Past history of hypertension, $\%$ & 13.4 & 13.5 & 13.2 \\
\hline Past history of diabetes, $\%$ & 7.2 & 4.1 & 2.8 \\
\hline Current smoker, \% & 5.4 & 4.7 & 6.4 \\
\hline Current alcohol consumption, \% & 19.5 & 18.1 & 17.9 \\
\hline Total energy, mean (SD), kcal/day & $1,660.7(483.2)$ & $1,915.6(548.4)$ & $1,928.7(567.7)$ \\
\hline Vegetables, mean (SD), g/day & $237.1(139.6)$ & $226.9(125.1)$ & $214.4(116.1)$ \\
\hline Fruit, mean (SD), g/day & $197.1(161.3)$ & $222.1(152.8)$ & $261.0(163.9)$ \\
\hline Coffee consumption, mean (SD), g/day & $107.1(143.1)$ & $99.4(138.1)$ & $97.8(131.4)$ \\
\hline
\end{tabular}

Table 1. Baseline characteristic of participants according to sugary drink consumption in Japanese men and women. $S D$ standard deviation, $B M I$ body mass index, $\mathrm{kg}$ kilogram, $m$ meter, $k c a l$ kilocalorie, $g$ gram. Sugary drinks included beta-carotene-fortified beverages, calcium-fortified beverages, canned coffee, carbonated beverages, $100 \%$ fruit juices (apple juice and orange juice), lactic acid bacteria beverages, and vitamin-fortified beverages.

potential carcinogenic effects of sugary drinks on bladder ${ }^{10}$. However, similar to kidney cancer, a positive association was observed in women after the exclusion of cancer cases diagnosed within the first three years of follow up. Therefore, the real associations in the primary analyses may be biased downward to null due to reverse causality.

This large population-based prospective study, to our knowledge, was the first attempt to assess the association of sugary drink consumption with risk of kidney and bladder cancer in the Western Pacific region, where the percentage increases in the incidence of kidney (117.1\%, 95\% UI 88.2-152.2\%) and bladder cancer (35\%, 95\% UI 17-59\%) were the highest and second highest, respectively, among World Health Organization regions between 1990 and $2019^{40}$. The major strength of this study is that the comprehensive lifestyle and dietary information were collected prospectively, with a high follow-up rate and long follow-up duration; this reduced the potential for recall bias and selection bias. Our study also has several limitations that warrant discussion. Owing to the self-reported nature of the questionnaire, misclassification from measurement error is inevitable. Given the prospective design of the study, however, such errors are likely to be non-differential. Nevertheless, we acknowledge that the correlation coefficients for validity were low in the cohort and that the use of fixed sugary drink items in the questionnaire might have resulted in imprecise measurement. As with any observational study, a possible impact of residual confounding could not be totally ruled out. Although we adjusted for major confounders that may have impacted the risk of kidney and bladder cancer, residual confounding from unmeasured variables, occupational, or socioeconomic factors is possible.

\section{Conclusions}

In summary, this large prospective cohort study demonstrated that sugary drink consumption was associated with slightly greater risks of kidney and bladder cancer in women, but not in men, after excluding incident cancers diagnosed in the first 3 years of follow-up. Additional studies with adequate cancer cases are needed to further examine the potential for reverse causation in this association. 


\begin{tabular}{|c|c|c|c|c|c|}
\hline & \multicolumn{3}{|c|}{ Category of sugary drink consumption, HR (95\% CI) } & $P$ value for trend & $\begin{array}{l}\text { Per } 100 \mathrm{ml} \text { per day } \\
\text { incremental, HR }(95 \% \mathrm{CI})\end{array}$ \\
\hline \multicolumn{6}{|l|}{ Kidney cancer } \\
\hline \multicolumn{6}{|l|}{ Both } \\
\hline Intake category, $\mathrm{ml} /$ day & Non-consumers & $\begin{array}{l}\text { Men: }>0-254 \\
\text { Female: }>0-134\end{array}$ & $\begin{array}{l}\text { Men: } \geq 254 \\
\text { Female: } \geq 134\end{array}$ & & \\
\hline No. of cases & 27 & 71 & 71 & & \\
\hline Person-years & 152,902 & 454,565 & 462,348 & & \\
\hline Minimally adjusted $^{\mathrm{a}}$ & 1 [reference] & $0.86(0.55-1.34)$ & $0.88(0.56-1.38)$ & 0.80 & $1.02(0.96-1.08)$ \\
\hline 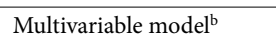 & 1 [reference] & $0.88(0.56-1.38)$ & $0.92(0.57-1.47)$ & 0.93 & $1.02(0.96-1.08)$ \\
\hline Excluding 3 years ${ }^{\mathrm{b}}$ & 1 [reference] & $0.90(0.54-1.50)$ & $1.08(0.64-1.81)$ & 0.56 & $1.04(0.99-1.10)$ \\
\hline Without HTN, DM, BMI & 1 [reference] & $0.88(0.56-1.38)$ & $0.92(0.57-1.46)$ & 0.92 & $1.02(0.96-1.08)$ \\
\hline \multicolumn{6}{|l|}{ Men } \\
\hline Intake category, $\mathrm{ml} /$ day & Non-consumers & $>0-254$ & $\geq 254$ & & \\
\hline No. of cases & 17 & 49 & 47 & & \\
\hline Person-years & 58,605 & 199,955 & 206,381 & & \\
\hline Minimally adjusted $^{\mathrm{a}}$ & 1 [reference] & $0.89(0.51-1.55)$ & $0.90(0.51-1.59)$ & 0.86 & $1.01(0.95-1.08)$ \\
\hline 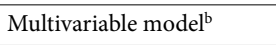 & 1 [reference] & $0.89(0.50-1.56)$ & $0.89(0.49-1.62)$ & 0.84 & $1.01(0.95-1.08)$ \\
\hline Excluding 3 years ${ }^{\mathrm{b}}$ & 1 [reference] & $0.82(0.44-1.50)$ & $0.94(0.50-1.76)$ & 0.78 & $1.03(0.96-1.09)$ \\
\hline Without HTN, DM, BMI & 1 [reference] & $0.90(0.51-1.58)$ & $0.91(0.50-1.64)$ & 0.87 & $1.01(0.95-1.08)$ \\
\hline \multicolumn{6}{|l|}{ Women } \\
\hline Intake category, $\mathrm{ml} /$ day & Non-consumers & $>0-134$ & $\geq 134$ & & \\
\hline No. of cases & 10 & 22 & 24 & & \\
\hline Person-years & 94,297 & 254,610 & 255,967 & & \\
\hline Minimally adjusted $^{\mathrm{a}}$ & 1 [reference] & $0.78(0.37-1.67)$ & $0.80(0.37-1.70)$ & 0.74 & $1.04(0.91-1.19)$ \\
\hline Multivariable model $^{\mathrm{b}}$ & 1 [reference] & $0.83(0.38-1.79)$ & $0.93(0.43-2.03)$ & 0.91 & $1.07(0.95-1.21)$ \\
\hline Excluding 3 years ${ }^{\mathrm{b}}$ & 1 [reference] & $1.06(0.41-2.72)$ & $1.37(0.54-3.50)$ & 0.37 & $1.11(1.01-1.22)$ \\
\hline Without HTN, DM, BMI & 1 [reference] & $0.81(0.38-1.75)$ & $0.91(0.42-1.98)$ & 0.95 & $1.07(0.95-1.20)$ \\
\hline \multicolumn{6}{|l|}{ Bladder cancer } \\
\hline \multicolumn{6}{|l|}{ Both } \\
\hline Intake category, $\mathrm{ml} /$ day & Non-consumers & $\begin{array}{l}\text { Men: }>0-254 \\
\text { Female: }>0-134\end{array}$ & $\begin{array}{l}\text { Men: } \geq 254 \\
\text { Female: } \geq 134\end{array}$ & & \\
\hline No. of cases & 50 & 126 & 121 & & \\
\hline Person-years & 152,902 & 454,565 & 462,348 & & \\
\hline Minimally adjusted $^{\mathrm{a}}$ & 1 [reference] & $0.84(0.60-1.17)$ & $0.85(0.61-1.20)$ & 0.57 & $1.02(0.98-1.06)$ \\
\hline Multivariable model $^{\mathrm{b}}$ & 1 [reference] & $0.81(0.58-1.13)$ & $0.79(0.56-1.12)$ & 0.33 & $1.01(0.97-1.06)$ \\
\hline Excluding 3 years ${ }^{\mathrm{b}}$ & 1 [reference] & $0.80(0.56-1.14)$ & $0.79(0.54-1.14)$ & 0.33 & $1.01(0.96-1.06)$ \\
\hline Without HTN, DM, BMI & 1 [reference] & $0.80(0.57-1.12)$ & $0.78(0.55-1.10)$ & 0.29 & $1.01(0.97-1.06)$ \\
\hline \multicolumn{6}{|l|}{ Men } \\
\hline Intake category, $\mathrm{ml} /$ day & Non-consumers & $>0-254$ & $\geq 254$ & & \\
\hline No. of cases & 40 & 96 & 93 & & \\
\hline Person-years & 58,605 & 199,955 & 206,381 & & \\
\hline Minimally adjusted $^{\mathrm{a}}$ & 1 [reference] & $0.74(0.51-1.07)$ & $0.75(0.51-1.09)$ & 0.37 & $1.01(0.96-1.05)$ \\
\hline Multivariable model $^{\mathrm{b}}$ & 1 [reference] & $0.73(0.50-1.06)$ & $0.73(0.49-1.08)$ & 0.34 & $1.01(0.96-1.05)$ \\
\hline Excluding 3 years ${ }^{\mathrm{b}}$ & 1 [reference] & $0.74(0.50-1.11)$ & $0.71(0.47-1.09)$ & 0.29 & $0.99(0.94-1.05)$ \\
\hline Without HTN, DM, BMI ${ }^{c}$ & 1 [reference] & $0.72(0.49-1.05)$ & $0.71(0.48-1.06)$ & 0.31 & $1.00(0.96-1.05)$ \\
\hline \multicolumn{6}{|l|}{ Women } \\
\hline Intake category, $\mathrm{ml} /$ day & Non-consumers & $>0-134$ & $\geq 134$ & & \\
\hline No. of cases & 10 & 30 & 28 & & \\
\hline Person-years & 94,297 & 254,610 & 255,967 & & \\
\hline Minimally adjusted $^{\mathrm{a}}$ & 1 [reference] & $1.25(0.61-2.58)$ & $1.28(0.61-2.67)$ & 0.64 & $1.10(1.01-1.20)$ \\
\hline 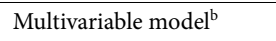 & 1 [reference] & $1.10(0.53-2.28)$ & $0.95(0.45-2.03)$ & 0.70 & $1.08(0.97-1.20)$ \\
\hline Excluding 3 years ${ }^{\mathrm{b}}$ & 1 [reference] & $0.96(0.44-2.10)$ & $1.01(0.46-2.23)$ & 0.89 & $1.11(1.01-1.22)$ \\
\hline Without HTN, DM, BMI & 1 [reference] & $1.11(0.53-2.31)$ & $0.97(0.46-2.06)$ & 0.74 & $1.08(0.97-1.20)$ \\
\hline
\end{tabular}


Table 2. Hazards for kidney and bladder cancer incidence according to intake of sugary drinks in Japanese men and women. HR hazard ratio, CI confidence interval, No. number. ${ }^{a}$ Minimally adjusted model adjusted for age (year); public health center (10 areas); smoking status and intensity (never; former; current: $<20$ cigarettes per day; current: $\geq 20$ cigarettes per day); physical activities (metabolic equivalent task, hours per day, tercile); and intake of total energy ( $\mathrm{kcal}$ per day) (continuous). ${ }^{\mathrm{b}}$ Multivariable Cox regression model adjusted for age (year); public health center (10 areas); body mass index $(<18.5,18.5$ to $<25,25$ to $<30$, or 30 to $<45$ ); history of hypertension (yes or no); history of diabetes (yes or no), smoking status and intensity (never; former; current: $<20$ cigarettes per day; current: $\geq 20$ cigarettes per day); consumption of alcohol (ethanol gram per week), coffee (gram per day), fruit (g per day, tercile), vegetables (g per day, tercile), biscuits (g per day, tercile), cake (g per day, tercile), chocolate (g per day, tercile), and Japanese-style confectionery (g per day, tercile); physical activities (metabolic equivalent task, hours per day, tercile); height (cm, tercile); and intake of total energy (kcal per day) (all continuous). ${ }^{c}$ History of hypertension, history of diabetes, and body mass index were not adjusted for. Sugary drinks included beta-carotene-fortified beverages, calcium-fortified beverages, canned coffee, carbonated beverages, $100 \%$ fruit juices (apple juice and orange juice), lactic acid bacteria beverages, and vitamin-fortified beverages.

\begin{tabular}{|c|c|c|c|c|}
\hline \multirow[b]{2}{*}{ Intake category, $\mathrm{ml} / \mathrm{day}$} & \multicolumn{3}{|c|}{ Category of sugary drink consumption, HR $(95 \% \mathrm{CI})$} & \multirow[b]{2}{*}{$P$ for interaction } \\
\hline & Non-consumers & $\begin{array}{l}\text { Men: }>0-254 \\
\text { Female: }>0-134\end{array}$ & $\begin{array}{l}\text { Men: } \geq 254 \\
\text { Female: } \geq 134\end{array}$ & \\
\hline \multicolumn{5}{|l|}{ Both sexes } \\
\hline \multicolumn{5}{|l|}{ Kidney cancer } \\
\hline \multicolumn{4}{|l|}{$\mathrm{BMI}^{\mathrm{a}}$} & 0.42 \\
\hline $\mathrm{BMI}<25$ & 1 [reference] & $0.99(0.56-1.74)$ & $0.90(0.50-1.64)$ & \\
\hline $\mathrm{BMI} \geq 25$ & 1 [reference] & $0.66(0.31-1.44)$ & $0.89(0.41-1.93)$ & \\
\hline \multicolumn{4}{|l|}{ History of hypertension ${ }^{\mathrm{b}}$} & 0.92 \\
\hline No & 1 [reference] & $0.82(0.49-1.37)$ & $0.90(0.53-1.53)$ & \\
\hline Yes & 1 [reference] & $1.04(0.39-2.75)$ & $0.88(0.31-2.50)$ & \\
\hline \multicolumn{4}{|l|}{ History of diabetes $^{c}$} & 0.79 \\
\hline No & 1 [reference] & $0.96(0.58-1.56)$ & $0.98(0.59-1.63)$ & \\
\hline Yes & 1 [reference] & $0.50(0.13-1.87)$ & $0.54(0.12-2.37)$ & \\
\hline \multicolumn{5}{|l|}{ Bladder cancer } \\
\hline \multicolumn{4}{|l|}{$\mathrm{BMI}^{\mathrm{a}}$} & 0.76 \\
\hline $\mathrm{BMI}<25$ & 1 [reference] & $0.77(0.51-1.15)$ & $0.77(0.51-1.17)$ & \\
\hline $\mathrm{BMI} \geq 25$ & 1 [reference] & $0.89(0.48-1.65)$ & $0.80(0.42-1.52)$ & \\
\hline \multicolumn{4}{|l|}{ History of hypertension ${ }^{\mathrm{b}}$} & 0.88 \\
\hline No & 1 [reference] & $0.80(0.55-1.16)$ & $0.78(0.53-1.15)$ & \\
\hline Yes & 1 [reference] & $0.86(0.40-1.84)$ & $0.83(0.37-1.87)$ & \\
\hline \multicolumn{4}{|l|}{ History of diabetes $^{c}$} & 0.70 \\
\hline No & 1 [reference] & $0.78(0.55-1.12)$ & $0.74(0.51-1.07)$ & \\
\hline Yes & 1 [reference] & $0.86(0.32-2.27)$ & $1.29(0.46-3.64)$ & \\
\hline
\end{tabular}

Table 3. Hazards for kidney and bladder cancer incidence according to intake of sugary drinks, stratified by body mass index, history of hypertension and diabetes. HR hazard ratio, CI confidence interval, No. number. Multivariable Cox regression model adjusted for age (year); public health center (10 areas); body mass index $(<18.5,18.5$ to $<25,25$ to $<30$, or 30 to $<45$ ); history of hypertension (yes or no); history of diabetes (yes or no), smoking status and intensity (never; former; current: $<20$ cigarettes per day; current: $\geq 20$ cigarettes per day); consumption of alcohol (ethanol gram per week), coffee (gram per day), fruit (g per day, tercile), vegetables (g per day, tercile), biscuits (g per day, tercile), cake (g per day, tercile), chocolate (g per day, tercile), and Japanese-style confectionery (g per day, tercile); physical activities (metabolic equivalent task, hours per

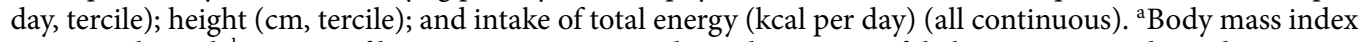
was not adjusted. ${ }^{b}$ History of hypertension was not adjusted. ${ }^{c}$ History of diabetes was not adjusted. Sugary drinks included beta-carotene-fortified beverages, calcium-fortified beverages, canned coffee, carbonated beverages, $100 \%$ fruit juices (apple juice and orange juice), lactic acid bacteria beverages, and vitamin-fortified beverages. 


\section{Data availability}

For information on how to apply for access to the JPHC data and/or biospecimens, please follow the instructions at http://epi.ncc.go.jp/en/jphc/805/8155.html.

Received: 18 January 2021; Accepted: 13 September 2021

Published online: 04 November 2021

\section{References}

1. James, S. L. et al. Global, regional, and national incidence, prevalence, and years lived with disability for 354 diseases and injuries for 195 countries and territories, 1990-2017: A systematic analysis for the Global Burden of Disease Study 2017. Lancet 392, 1789-1858 (2018).

2. Bray, F. et al. Global cancer statistics 2018: GLOBOCAN estimates of incidence and mortality worldwide for 36 cancers in 185 countries. CA Cancer J. Clin. 68, 394-424 (2018).

3. World Cancer Research Fund/American Institute for Cancer Research. Continuous Update Project Expert Report 2018. Diet, Nutrition, Physical Activity and Kidney Cancer. https://www.wcrf.org/dietandcancer. Accessed 25 Oct 2020.

4. World Cancer Research Fund/American Institute for Cancer Research. Continuous Update Project Expert Report 2018. Diet, Nutrition, Physical Activity and Bladder Cancer. https://www.wcrf.org/dietandcancer. Accessed 25 Oct 2020.

5. Chazelas, E. et al. Sugary drink consumption and risk of cancer: results from NutriNet-Santé prospective cohort. BMJ 366, 12408 (2019).

6. Rebholz, C. M. et al. Patterns of beverages consumed and risk of incident kidney disease. Clin. J. Am. Soc. Nephrol. 14, 49-56 (2019).

7. Lowrance, W. T., Ordoñez, J., Udaltsova, N., Russo, P. \& Go, A. S. CKD and the risk of incident cancer. J. Am. Soc. Nephrol. 25, 2327-2334 (2014).

8. Malik, V. S., Popkin, B. M., Bray, G. A., Després, J. P. \& Hu, F. B. Sugar-sweetened beverages, obesity, type 2 diabetes mellitus, and cardiovascular disease risk. Circulation 121, 1356-1364 (2010).

9. Graff, R. E. et al. Type 2 diabetes in relation to the risk of renal cell carcinoma among men and women in two large prospective cohort studies. Diabetes Care 41, 1432-1437 (2018).

10. Braver, D. J., Modan, M., Chêtrit, A., Lusky, A. \& Braf, Z. Drinking, micturition habits, and urine concentration as potential risk factors in urinary bladder cancer. J. Natl. Cancer Inst. 78, 437-440 (1987).

11. International Agency for Research on Cancer. IARC Monographs on the Evaluation of Carcinogenic Risk to Humans, vol. 101. (International Agency for Research on Cancer, 2013). https://publications.iarc.fr/Book-And-Report-Series Accessed 12 Nov 2020.

12. Zhu, H. et al. Carbohydrates, glycemic index, and glycemic load in relation to bladder cancer risk. Front. Oncol. 10, 1838 (2020).

13. Hodge, A. M., Bassett, J. K., Milne, R. L., English, D. R. \& Giles, G. G. Consumption of sugar-sweetened and artificially sweetened soft drinks and risk of obesity-related cancers. Public Health Nutr. 21, 1618-1626 (2018).

14. Lee, J. E. et al. Intakes of coffee, tea, milk, soda and juice and renal cell cancer in a pooled analysis of 13 prospective studies. Int. J. Cancer 121, 2246-2253 (2007).

15. Ros, M. M. et al. Fluid intake and the risk of urothelial cell carcinomas in the European Prospective Investigation into Cancer and Nutrition (EPIC). Int. J. Cancer 128, 2695-2708 (2011).

16. Michaud, D. S. et al. Fluid intake and the risk of bladder cancer in men. N. Engl. J. Med. 340, 1390-1397 (1999).

17. Sasaki, S., Kobayashi, M., Ishihara, J. \& Tsugane, S. Self-administered food frequency questionnaire used in the 5-year follow-up survey of the JPHC Study: Questionnaire structure, computation algorithms, and area-based mean intake. J. Epidemiol. 13, S13-S22 (2003).

18. Ishihara, J. et al. Impact of the revision of a nutrient database on the validity of a self-administered food frequency questionnaire (FFQ). J. Epidemiol. 16, 107-116 (2006).

19. Huang, H. L. et al. Association of sugary drink consumption with all-cause and cause-specific mortality: The Japan Public Health Center-based Prospective Study. Prev. Med. 148, 106561 (2021).

20. Leung, C. Y. et al. Sugary drink consumption and subsequent colorectal cancer risk: The Japan Public Health Center-based prospective cohort study. Cancer Epidemiol. Biomarkers Prev. 30, 782-788 (2021).

21. Alina, M. H. \& Alexandru, M. G. Diet, Microbiome and health, vol. 11. Handbook of Food Bioengineering, Chapter 4 (Academic Press, 2018).

22. World Health Organization. International Classification of Diseases for Oncology, 3rd ed. (World Health Organization, 2000).

23. Larsson, S. C. \& Wolk, A. Diabetes mellitus and incidence of kidney cancer: A meta-analysis of cohort studies. Diabetologia 54, 1013-1018 (2011)

24. Inoue, M. Impact of lifestyle on overall cancer risk among Japanese: The Japan Public Health Center-based Prospective Study (JPHC Study). J. Epidemiol. 20, 90-96 (2010).

25. Larsson, S. C., Orsini, N., Brismar, K. \& Wolk, A. Diabetes mellitus and risk of bladder cancer: A meta-analysis. Diabetologia 49, 2819-2823 (2006).

26. Sawada, N. et al. Body mass index and subsequent risk of kidney cancer: A prospective cohort study in Japan. Ann. Epidemiol. 20, 466-472 (2010).

27. Kim, C. S. et al. Association of hypertension and blood pressure with kidney cancer risk: A nationwide population-based cohort study. Hypertension 75, 1439-1446 (2020).

28. Cumberbatch, M. G., Rota, M., Catto, J. W. \& La Vecchia, C. The role of tobacco smoke in bladder and kidney carcinogenesis: A comparison of exposures and meta-analysis of incidence and mortality risks. Eur. Urol. 70, 458-466 (2016).

29. Masaoka, H. et al. Alcohol consumption and bladder cancer risk with or without the flushing response: The Japan Public Health Center-based Prospective Study. Int. J. Cancer 141, 2480-2488 (2017).

30. Kurahashi, N., Inoue, M., Iwasaki, M., Sasazuki, S. \& Tsugane, S. Coffee, green tea, and caffeine consumption and subsequent risk of bladder cancer in relation to smoking status: A prospective study in Japan. Cancer Sci. 100, 284-291 (2009).

31. Durrleman, S. \& Simon, R. Flexible regression models with cubic splines. Stat. Med. 8, 551-561 (1989).

32. Feig, D. I., Kang, D. H. \& Johnson, R. J. Uric acid and cardiovascular risk. N. Engl. J. Med. 359, 1811-1821 (2008).

33. Jung, S. W., Kim, S. M., Kim, Y. G., Lee, S. H. \& Moon, J. Y. Uric acid and inflammation in kidney disease. Am. J. Physiol. Renal Physiol. 318, F1327-1340 (2020).

34. Bavishi, C., Messerli, F. H. \& Rimoldi, S. F. Serum uric acid in primary hypertension: From innocent bystander to primum movens?. Hypertension 67, 845-847 (2016).

35. Bellomo, G. et al. Association of uric acid with change in kidney function in healthy normotensive individuals. Am. J. Kidney Dis. 56, 264-272 (2010).

36. Mwasongwe, S. E. et al. Relation of uric acid level to rapid kidney function decline and development of kidney disease: The Jackson Heart Study. J. Clin. Hypertens. 20, 775-783 (2018).

37. Muntner, P. et al. The prevalence of nontraditional risk factors for coronary heart disease in patients with chronic kidney disease. Ann. Intern. Med. 140, 9-17 (2004) 
38. Shlipak, M. G. et al. Elevations of inflammatory and procoagulant biomarkers in elderly persons with renal insufficiency. Circulation 107, 87-92 (2003).

39. Coussens, L. M. \& Werb, Z. Inflammation and cancer. Nature 420, 860-867 (2002).

40. Institute for Health Metrics and Evaluation (IHME). GBD Compare. (IHME, University of Washington, 2015). http://vizhub.healt hdata.org/gbd-compare. Accessed 18 May 2021.

\section{Acknowledgements}

The JPHC Study Group members are listed at the following site (as of October, 2020): https://epi.ncc.go.jp/en/ jphc/781/8390.html. The authors would like to thank Rieko Kanehara and Dr. Utako Murai of the Epidemiology and Prevention Group, Center for Public Health Sciences, National Cancer Center, Tokyo, Japan for their advice on the nutritional aspects of the study.

\section{Author contributions}

M. Inoue and C.Y.L. designed the study. C.Y.L. performed the analysis and wrote the manuscript. C.Y.L., S.K.A., N.S., J.I., R.T., T.Y., M.I., M.H., M. Inoue, and S.T. revised the manuscript critically for important intellectual content. All authors approved the final version before submission.

\section{Funding}

This study was supported by the National Cancer Centre Research and Development Fund (Since 2011), and a Grant in Aid for Cancer Research from the Ministry of Health, Labour and Welfare of Japan (from 1989 to 2010).

\section{Competing interests}

The authors declare no competing interests.

\section{Additional information}

Supplementary Information The online version contains supplementary material available at https:/doi.org/ 10.1038/s41598-021-01103-x.

Correspondence and requests for materials should be addressed to M.I.

Reprints and permissions information is available at www.nature.com/reprints.

Publisher's note Springer Nature remains neutral with regard to jurisdictional claims in published maps and institutional affiliations.

(c) (i) Open Access This article is licensed under a Creative Commons Attribution 4.0 International License, which permits use, sharing, adaptation, distribution and reproduction in any medium or format, as long as you give appropriate credit to the original author(s) and the source, provide a link to the Creative Commons licence, and indicate if changes were made. The images or other third party material in this article are included in the article's Creative Commons licence, unless indicated otherwise in a credit line to the material. If material is not included in the article's Creative Commons licence and your intended use is not permitted by statutory regulation or exceeds the permitted use, you will need to obtain permission directly from the copyright holder. To view a copy of this licence, visit http://creativecommons.org/licenses/by/4.0/.

(C) The Author(s) 2021 